matrix that couple the $267 \mathrm{~nm}$ transitions, and through the 260-267nm off-diagonal elements. It is therefore important that the $267 \mathrm{~nm}$ and $260 \mathrm{~nm}$ transitions should be treated simultaneously and not in isolation.

Two regions have been found in which the properties of the model resemble those of App5'A. These are characterized by a stacking angle close to $0^{\circ}$ and one close to $90^{\circ}$. In all of these the circulardichroism profile derives from the overlap of all four transitions. With $\alpha \approx 0^{\circ}$ the main contributors are one of the $267 \mathrm{~nm}$ transitions and a $260 \mathrm{~nm}$ transition that has been shifted by the complex exciton interactions to approx. $265 \mathrm{~nm}$. With $\alpha \approx 90^{\circ}$ the profile derives from the resultant of two strong opposite bands close to $260 \mathrm{~nm}$ with some rotational strength at $267 \mathrm{~nm}$. Of the two solutions the one with the low stacking angle would appear to be favoured, since it is the better candidate for the generation of strong hypochromism, as is shown by App5'A. This inference will be tested by including interactions with transitions located at $200 \mathrm{~nm}$.

Bayley, P. M. (1968). Abstr. I.U.P.A.C. 5th int. Symp., London, no. E23.

Bush, C. A. \& Tinoco, I., jun. (1967). J. molec. Biol. 23, 601.

Clark, L. B. \& Tinoco, I., jun. (1965). J. Am. chem. Soc. 87, 11.

Davis, R. C. \& Tinoco, I., jun. (1968). Biopolymers, 6, 223.

Massoulie, J. \& Michelson, A. M. (1964). C. r. hebd. Séanc. Acad. Sci., Paris, 259, 2923.

Michelson, A. M. (1963). The Chemistry of Nucleosides and Nucleotides, p. 447. New York: Academic Press Inc.

Schneider, A. S. \& Harris, R. A. (1969). J. chem. Phys. 50, 5204.

Scott, J. F. \& Zameenik, P. C. (1969). Proc. natn. Acad. Sci. U.S.A. 64, 1308.

\section{A Refinement of the Structure of Lysozyme}

By R. Diamond and M. Levitt. (Medical Research Council Laboratory of Molecular Biology, Hills Road, Cambridge CB2 2QH, U.K.)

Two techniques will be outlined. The first technique (due to R.D.) optimizes the interpretation of an electron-density map in terms of a flexible-chain molecule, and the second technique (due to M.L.) minimizes an energy function associated with the molecule. Collaborative use of the two techniques leads at once to co-ordinates that are stable with respect to both procedures to better than $0.1 \AA$ root mean square. Remaining problems that will be discussed are concerned with the best treatment of indistinct regions of the electrondensity map and with the empirical improvement of the energy parameters used in the second technique.

\section{The Molecular Structure of Hyaluronic Acid}

By E. D. T. Atkins and J. K. Sheehan. (H. $H$. Wills Physics Laboratory, University of Bristol, Royal Fort, Tyndall Avenue, Bristol BS8 1TL, U.K.)

Hyaluronic acid exists naturally on a hydrated gel. It is the constituent of synovial fluid, which has the property of acting as a lubricant; it also occurs in the vitreous humour, where its function is probably to maintain the precise shape of the eye. Hyaluronic acid is a member of a small group of biologically important macromolecules known as the mucopolysaccharides. Chemically it is a regular unbranched polymer with a repeating unit

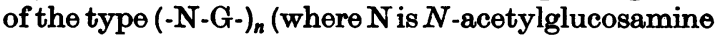
and $\mathrm{G}$ is glucuronic acid). The glycosidic linkages between $N$-acetylglucosamine and glucuronic acid and between glucuronic acid and $N$-acetylglucosamine are (1eq,4eq) and (leq,3eq) respectively.

Recently we have obtained a number of oriented fibres and films of hyaluronic acid together with its salts with various univalent and bivalent ions.

From the X-ray fibre-type diffraction patterns obtained we have been able to elucidate the molecular structure of the free acid and its salts. Sodium hyaluronate, for example, may be crystallized in two distinct hexagonal unit cells, but in each case the molecular conformation of an individual chain is a threefold helix. In the free acid the conformation is tightened to a twofold helix.

We also suggest a reason for the alternate $(1 e q, 3 e q)$ and $(1 e q, 4 e q)$ glycosidic linkages. They are needed to position all the important substituent groups equally on both sides of the chain in the free acid form. The simpler $(1 \rightarrow 4)$-linked hexosans [e.g. chitin (Carlstrom, 1957) and polymannuronic acid (Atkins, Mackie \& Smolko, 1970)] need only one type of linkage to acquire the same features. On formation of a salt with a univalent ion a rotation occurs along the chain; this is partly due to steric hindrance of the cation but also has the advantage of distributing the ionic charge more evenly over the surface of the polymer.

The ability to crystallize the material in a number of forms enabling the chain conformation to be sampled in a variety of environments will help in predicting the molecular shape in the more dilute environment.

Atkins, E. D. T., Mackie, W. \& Smolko, E. E. (1970). Nature, Lond., 225, 626.

Carlstrom, D. (1957). J. biophys. biochem. Cytol. 3, 669. 